\title{
A first-principles theoretical study of the electronic and optical properties of twisted bilayer GaN structures
}

\author{
Xiang Cai ${ }^{1}$. Shuo Deng ${ }^{2} \cdot \mathrm{Lijie}^{1} \mathrm{Li}^{1} \cdot \mathrm{Ling} \mathrm{Hao}^{3}$
}

Published online: 11 May 2020

(c) The Author(s) 2020

\begin{abstract}
Gallium nitride $(\mathrm{GaN})$ is a well-investigated material that is applied in many advanced power electronic and optoelectronic devices due to its wide bandgap. However, derivatives of its monolayer form, such as bilayer structures, have rarely been reported. We study herein the electronic and optical properties of $\mathrm{GaN}$ bilayer structures that are rotated in the plane at several optimized angles by using the density functional theory method. To maintain the structural stability and use a small cell size, the twisting angles of the GaN bilayer structures are optimized to be $27.8^{\circ}, 38.2^{\circ}$, and $46.8^{\circ}$ using the crystal matching theory. The band-structure analysis reveals that the bandgap is wider for the twisted structures compared with the nontwisted case. The simulation results provide the absorption coefficient, extinction coefficient, reflectivity, and refractive index at these angles. The spectra of all these optical properties match with the bandgap values. The simulated refractive index of the bilayer structures at all the twisting angles including $0^{\circ}$ is smaller than that of bulk $\mathrm{GaN}$, indicating a reduced scattering loss for optoelectronics applications. Considering the results of this analysis, the possible applications may include low-loss integrated electronic and optical devices and systems.
\end{abstract}

Keywords GaN bilayer · Twisting effect $\cdot$ Optical properties

\section{Introduction}

$\mathrm{GaN}$ is a typical group III-V compound semiconductor material and has been widely applied in power semiconductor and optoelectronic devices. For optical applications, $\mathrm{GaN}$ can be used as a substrate to make violet (405 nm) laser diodes. Light-emitting diodes (LEDs) made using GaN can achieve high brightness and cover the range of primary colors, being applicable in daylight-visible full-color LED displays and white LEDs. Because of their low sensitivity to ionizing radiation, GaN-based solar cell arrays are suitable for use on satellites. Meanwhile, the working temperature

Lijie Li

L.Li@swansea.ac.uk

Ling Hao

ling.hao@npl.co.uk

1 College of Engineering, Swansea University, Swansea SA1 8EN, UK

2 School of Electronic and Electrical Engineering, Nanyang Technological University, Singapore, Singapore

3 Quantum Technologies Department, National Physics Laboratory, Teddington TW11 0LW, UK and voltage limit of $\mathrm{GaN}$ transistors are higher than those of gallium arsenide (GaAs) transistors, leading to many applications in microwave power amplifier devices [1-4]. GaN has also become one of the important materials for use in next-generation smartphones and Internet technologies, as it can meet the requirements of high power, high temperature, low refractive index ( 2.3 for $\mathrm{GaN}$ compared with 3.5 for $\mathrm{GaAs}$ ), and superior temperature stability of the refractive index (one order of magnitude higher than that of InP).

It was reported in past studies that devices with a suspended bilayer structure offer advantages in terms of enhanced electronic performance. Indeed, such two-dimensional (2D) material forms are deemed to have much thinner thickness than the bulk form, resulting in different electronic, mechanical, and optical performance characteristics [5-15]. This is due to the change of the crystallization and the quantum effects in 2D materials. Some previous work applying density functional theory to investigate the electronic properties of 2D materials has been conducted [16]. First-principles simulations of $\mathrm{GaN}$ as a single-layer III-V binary compound semiconductor have also been reported [17]. The band structures of single-layer $\mathrm{GaN}$ can be controlled via chemical functionalization $[18,19]$, adsorption 
of nonmetal materials [20-23], vacancies [24, 25], and doping $[26,27]$ methods. These results have indicated that 2D GaN has potential as a future developmental trend for high-performance nanoelectronic devices. Experimentally, it has been reported $[28,29]$ that $2 \mathrm{D} \mathrm{GaN}$ can be synthesized by the migration-enhanced encapsulated growth (MEEG) technique utilizing epitaxial graphene. It can also be grown by using the surface-confined nitridation reaction method on liquid metals [30], demonstrating the potential to implement 2D GaN in real nanoelectronic devices [23]. Structures formed by stacking two single-layer materials via van der Waals (vdW) forces and rotating one of them against the other may display interesting characteristics. The first studies in this regard $[31,32]$ reported that the use of a particular rotating/twisting angle in bilayer graphene resulted in superior electrical conductivity. In that work, the twisting angle could be controlled by using graphene produced by the exfoliation method. These previous papers on the twisting effect in stacked 2D materials have stimulated researchers to conduct studies on other multilayer structures based on various monolayer materials, including heterostructures. Specifically, for 2D GaN, such investigations have been performed using the first-principles method to investigate the effect of twisting in bilayer GaN. The twisted GaN bilayer model is built and subsequently analyzed herein to reveal its electronic and optical properties. These results will lead to wider and deeper explorations of single-layer $\mathrm{GaN}$ in the hope its application in practical electronic and optoelectronic devices.

\section{Computational methods}

The modeling of the bilayer GaN structure is based on the Quantum Atomistix ToolKit simulation tools. The simulations are conducted using density functional theory (DFT). The method of generalized gradient approximation (GGA) with the Perdew-Burke-Ernzerhof (PBE) parameterization at a temperature of $300 \mathrm{~K}$ with a mesh cutoff energy of 50 Hartrees and an $11 \times 11 \times 1 \mathbf{k}$-point grid are used to optimize all the structures. Considering the influence of the vdW force applied to the bilayers, semiempirical corrections using the Grimme DFT-D2 model are applied to include the longrange van der Waals interaction. A large (>20 A) vacuum spacing is added to the structure along the $c$-direction to avoid the application of periodic boundary conditions in this direction. The maximum force on each atom is set to $0.01 \mathrm{eV} / \AA$ for the structural relaxation calculations. For the simulation of the electronic and optical properties, the TB09 meta-GGA method is used for the bandgap, projected density of states, and optical properties with a $c$ parameter of 1.76. Figure 1 shows the calculated $c$ parameters against the calculated bandgap. The $c$ value is calculated to match

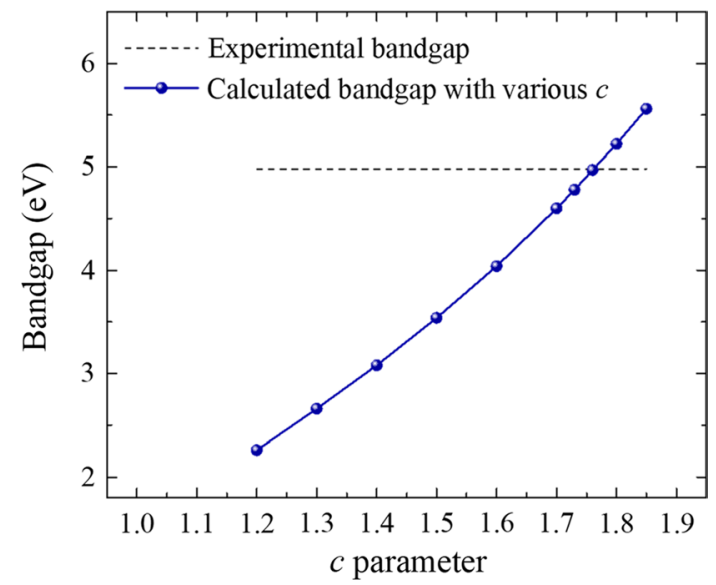

Fig. 1 The bandgap versus the $c$ parameter

the experimental bandgap of $4.98 \mathrm{eV}$ reported previously [28] for (untwisted) two-monolayer structures. There are no reports to date on the bandgap for two vdW-bonded $\mathrm{GaN}$ monolayers. The vdW bonding has less effect on the bandgap of the monolayer structure. It is reported that the $\mathrm{vdW}$ interaction does not affect the properties close to the Fermi level [33] for vdW-bonded heterostructures, indicating that the bandgap of the vdW-bonded GaN bilayer should be similar to that of the monolayer. Side views of the GaN bilayer structure are shown in Fig. 2. From this viewpoint, the structure is built by stacking two layers of a 2D GaN cell. Figure 2 also shows a bottom view of the different rotated structures. The rotation angles are selected according to the criteria of optimized stress and atom number, with the aim of achieving the most stable structures. The method of selecting optimal rotation angles was described previously [34]. Generally speaking, for the twisted bilayer structure, the number of atoms in a single cell increases as the rotation angle is increased. Table 1 presents the number of atoms and bandgap in each structure. However, when the angle equals $38.21^{\circ}$, the atom number decreases to 56 , much less than for the rotation angles of $27.8^{\circ}$ and $46.8^{\circ}$. All the models are passivated with hydrogen atoms. Figure 3 shows the band structures of the atomic model and the projected density of states (PDOS) for the bilayer GaN structures with the four rotation angles. The bilayer structures have bandgaps of $4.97 \mathrm{eV}, 5.06 \mathrm{eV}, 5.25 \mathrm{eV}$, and $5.22 \mathrm{eV}$ for the angles of $0^{\circ}$, $27.8^{\circ}, 38.2^{\circ}$, and $46.8^{\circ}$, respectively, indicating that bilayer $\mathrm{GaN}$ displays a typical wide-bandgap semiconducting property. The bandgap values are significantly larger than the value for bulk GaN. For all the structures, both the valenceband maximum (VBM) and the conduction-band minimum (CBM) occur at the $\Gamma$ point, indicating that the bandgap is of direct type. According to the PDOS results, the VBM is primarily composed of $\mathrm{N} 2 p$ and $\mathrm{H} 1 s$ orbitals while the $\mathrm{CBM}$ is a hybridization of the Ga $3 s, \mathrm{Ga} 3 p, \mathrm{~N} 2 s$, and $\mathrm{H}$ 

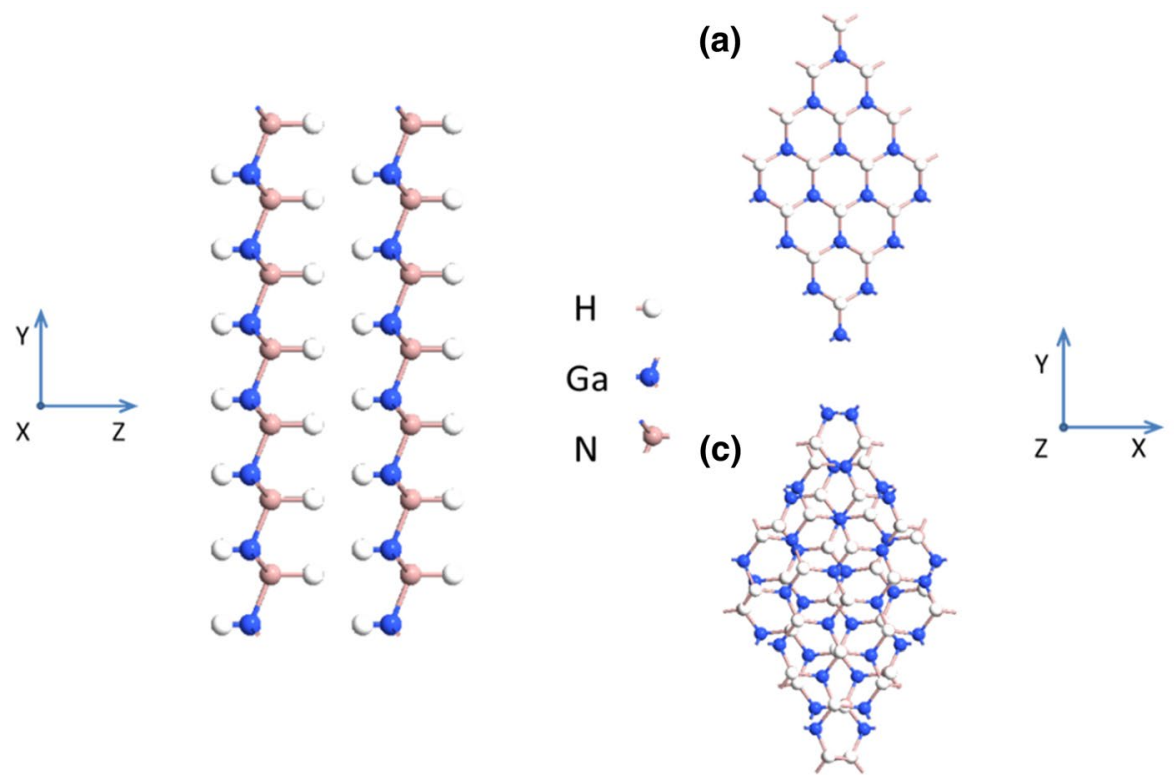

(b)

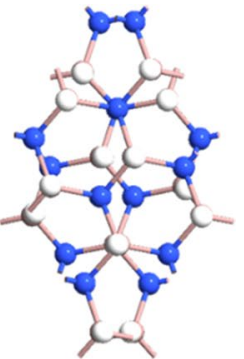

(d)

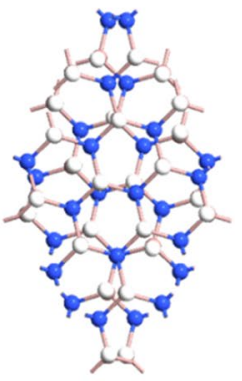

Fig. 2 A side view of the rotated structure, and bottom views of the structure with rotation of $\mathbf{a} 0^{\circ}, \mathbf{b} 38.2^{\circ}, \mathbf{c} 27.8^{\circ}$, and $\mathbf{d} 46.8^{\circ}$

Table 1 The atom numbers and bandgap value of the different rotated structure

\begin{tabular}{lllll}
\hline Rotation $\left(^{\circ}\right)$ & 0 & 27.8 & 38.21 & 46.83 \\
Atom numbers in single cell & 8 & 104 & 56 & 152 \\
Angle between vectors $\left(^{\circ}\right)$ & 60 & 60 & 60 & 60 \\
Bandgap (eV) & 4.97 & 5.06 & 5.25 & 5.22 \\
\hline
\end{tabular}

$1 s$ orbitals, although Ga $3 p$ contributes the most. The CBM occurs in Ga $3 p$ orbitals while the VBM occurs in N $2 p$. Figure 4 displays the results for the bandgap, CBM, and VBM versus the rotation angle. As the rotation angle is increased, the bandgap first increases (from $0^{\circ}$ to $38.2^{\circ}$ ) then decreases slightly (from $38.2^{\circ}$ to $46.8^{\circ}$ ). The $\mathrm{CBM}$ and $\mathrm{VBM}$ remain at the $\mathrm{G}$ point with increasing rotation angle. However, when
Fig. 3 The bandgap and PDOS of the structure with rotation of $\mathbf{a} 0^{\circ}, \mathbf{b} 27.8^{\circ}, \mathbf{c} 38.2^{\circ}$, and d $46.8^{\circ}$
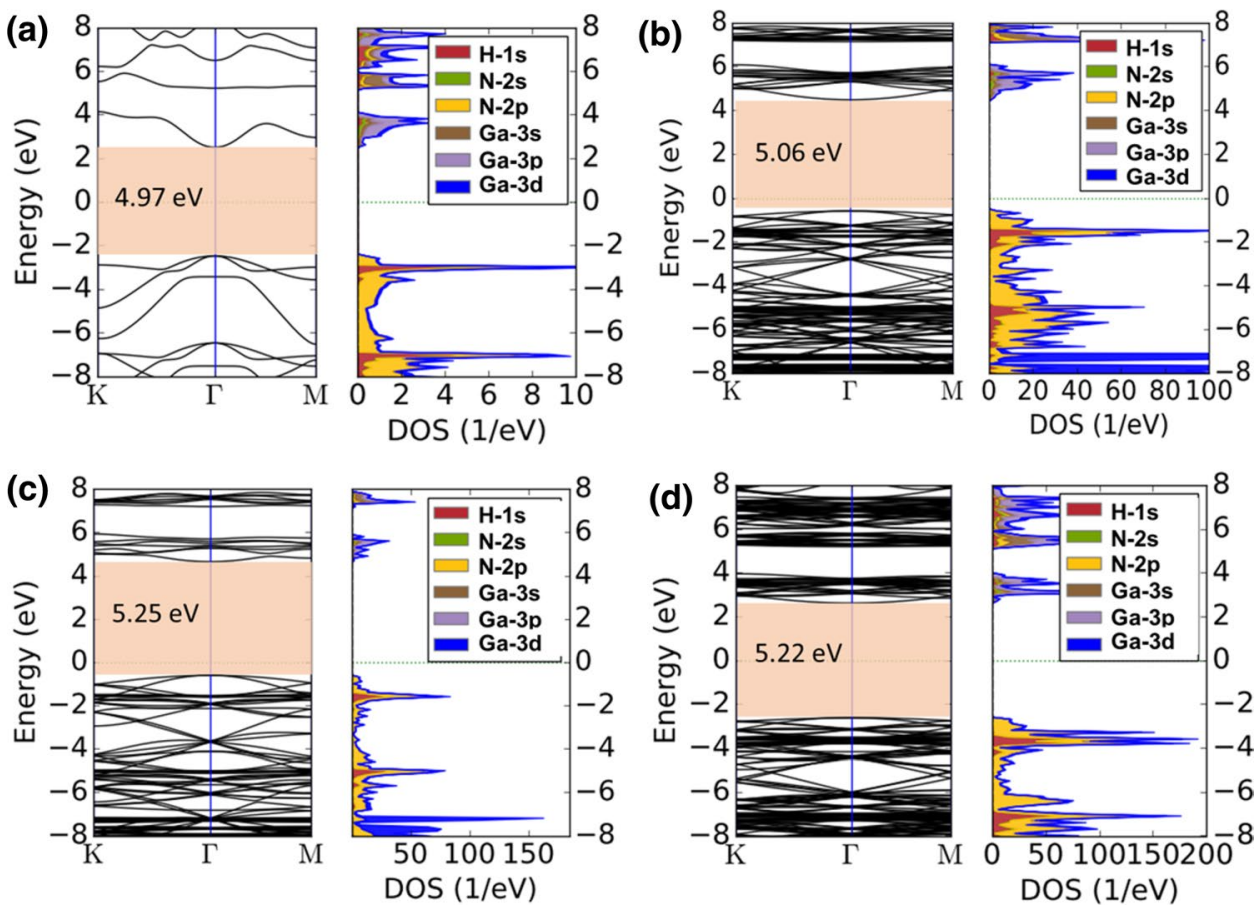
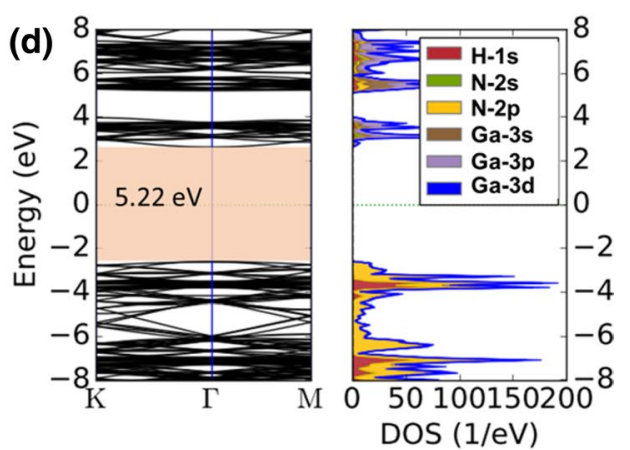


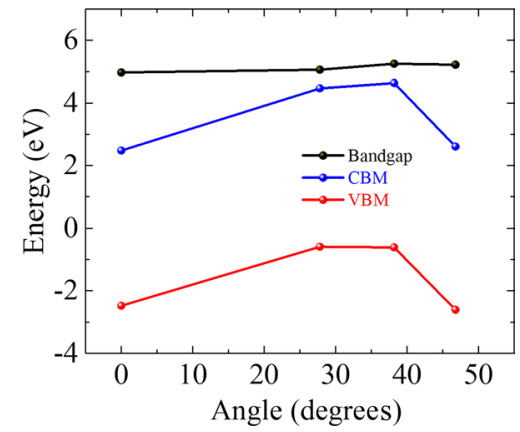

Fig. 4 The bandgap, CBM, and CBM versus the rotation angle

the angle is $27.8^{\circ}$ or $38.2^{\circ}$, the absolute value of the CBM is larger than that of VBM, indicating that the Fermi level moves towards the valence band.

\section{Optical properties}

The bilayer GaN models are simulated to determine several key optical properties such as the absorption coefficient and refractive index using the general mathematical procedure described in our previous work [35]. The susceptibility tensor at a frequency $\omega$, which characterizes the polarization of a material under an electrical field, can be written using the Kubo-Greenwood formula as

$\chi_{i j}(\omega)=-\frac{e^{2} \hbar^{4}}{m^{2} \varepsilon_{0} V \omega^{2}} \sum_{n m} \frac{f\left(E_{m}\right)-f\left(E_{n}\right)}{E_{n m}-\hbar \omega-i \Gamma} \pi_{n m}^{i} \pi_{m n}^{j}$,

where $E_{n}, E_{m}$, and $E_{n m}$ represent the energy levels at the energy states $n$ and $m$ and the energy difference between $n$ and $m . f \Gamma$ and $V$ stand for the Fermi function broadening and volume, respectively. $\pi_{n m}^{i}$ is the $i$ th component of the dipole matrix element between the states $n$ and $m . \varepsilon_{0}$, $\hbar$, and $e$ represent the permittivity in vacuum, the reduced Planck's constant, and the single electron charge, respectively. The susceptibility tensor is used to derive the relative permittivity $\left(\varepsilon_{\mathrm{r}}\right)$, which determines the amount of polarization of a dielectric material under an external electric field. It is

$\varepsilon_{\mathrm{r}}(\omega)=(1+\chi(\omega))$.

The polarization response of a material such as $\mathrm{GaN}$ under an electric field should be expressed in complex form, as there is always a phase change of the electric field. A simple expression for the complex permittivity at a frequency $\omega$ is

$\varepsilon_{\mathrm{r}}(\omega)=\varepsilon_{0} \varepsilon_{\mathrm{r}}(\omega)=\varepsilon^{\prime}(\omega)+\mathrm{i} \varepsilon^{\prime \prime}(\omega)$,

where $\varepsilon_{0}, \varepsilon^{\prime}$, and $\varepsilon^{\prime \prime}$ are the vacuum permittivity and the real and imaginary parts of the complex permittivity, respectively. Further expressions for the complex terms of the permittivity can be obtained from the Kramers-Kronig relations (bidirectional mathematical relations that connect the real and imaginary parts of any complex function) as follows:

$\varepsilon^{\prime}(\omega)=1+\frac{2}{\pi} A \int_{0}^{\infty} \frac{\omega^{\prime} \varepsilon^{\prime \prime}\left(\omega^{\prime}\right)}{\omega^{\prime 2}-\omega^{2}} \mathrm{~d} \omega^{\prime}$,

$\varepsilon^{\prime \prime}(\omega)=-\frac{2 \omega}{\pi} A \int_{0}^{\infty} \frac{\varepsilon^{\prime}\left(\omega^{\prime}\right)}{\omega^{\prime 2}-\omega^{2}} \mathrm{~d} \omega^{\prime}+\frac{\sigma_{0}}{\varepsilon_{0} \omega}$,

where $\sigma_{0}$ and $A$ denote the direct-current (DC) conductivity and the Cauchy principal value. The refractive index $(n)$ and the extinction coefficient $(\kappa)$ are related to $\varepsilon(\omega)$ by the relation $\varepsilon(\omega)=n+\mathrm{i} \kappa$. Hence from Eqs. (4) and (5), the refractive index $(n)$ and the extinction coefficient $(\kappa)$ can be written as

$n=\sqrt{\frac{\sqrt{\varepsilon^{\prime 2}+\varepsilon^{\prime \prime 2}}+\varepsilon^{\prime}}{2},}$

$\kappa=\sqrt{\frac{\sqrt{\sqrt{\varepsilon^{\prime 2}+\varepsilon^{\prime \prime 2}}}-\varepsilon^{\prime \prime}}{2}}$.

The optical absorption coefficient $\left(\alpha_{\mathrm{a}}\right)$ can then be written as

$\alpha_{\mathrm{a}}=2 \frac{\omega}{c} \kappa$,

where $c$ is the speed of light and $\omega$ is the angular frequency of the light. The reflectivity $(r)$ is expressed as

$r=\frac{(1-n)^{2}+\kappa^{2}}{(1+n)^{2}+\kappa^{2}}$.

Figure 5 depicts the real and imaginary parts of the complex permittivity calculated for the rotated bilayer $\mathrm{GaN}$ structures for twisting angles of $0^{\circ}, 27.8^{\circ}, 38.2^{\circ}$, and $46.8^{\circ}$. In terms of the amplitude, the values are similar in all cases, except that the permittivity shows maximum peak values when the rotation angle is $38.2^{\circ}$. The peak wavelengths in all cases coincide with the bandgap values; i.e., there is a slight blue-shift for nonzero twisting angles. In Fig. 5a, the real part of the permittivity at a long wavelength of $400 \mathrm{~nm}$ is $1.7322,1.7323,1.7322$, and 1.7322 for rotation angles of $0^{\circ}, 27.8^{\circ}, 38.2^{\circ}$, and $46.8^{\circ}$, respectively. The peak values of the imaginary permittivity appear between wavelengths of $200 \mathrm{~nm}$ and $250 \mathrm{~nm}$ in all cases according to Fig. 5b. Figure 6 shows the reflectivity, refractive index, absorption coefficient, and extinction coefficient spectra for the rotation angles of $0^{\circ}, 27.8^{\circ}, 38.2^{\circ}$, and $46.8^{\circ}$. Figure 6 a shows the reflectivity curves against wavelength, being very similar in 
Fig. 5 a The real and $\mathbf{b}$ imaginary parts of the permittivity
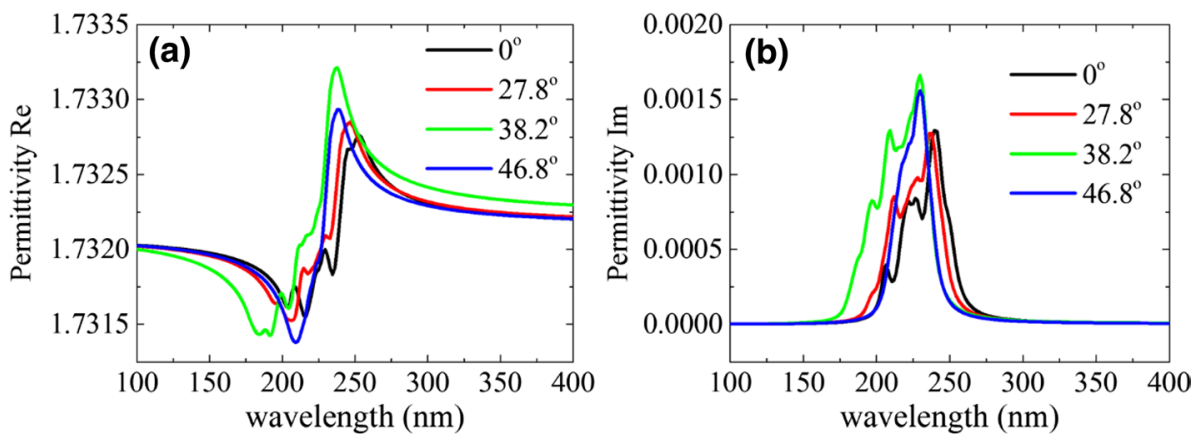

Fig. 6 The a reflectivity, b refractive index, $\mathbf{c}$ absorption coefficient, and $\mathbf{d}$ extinction coefficient spectra of the rotated bilayer $\mathrm{GaN}$ structures
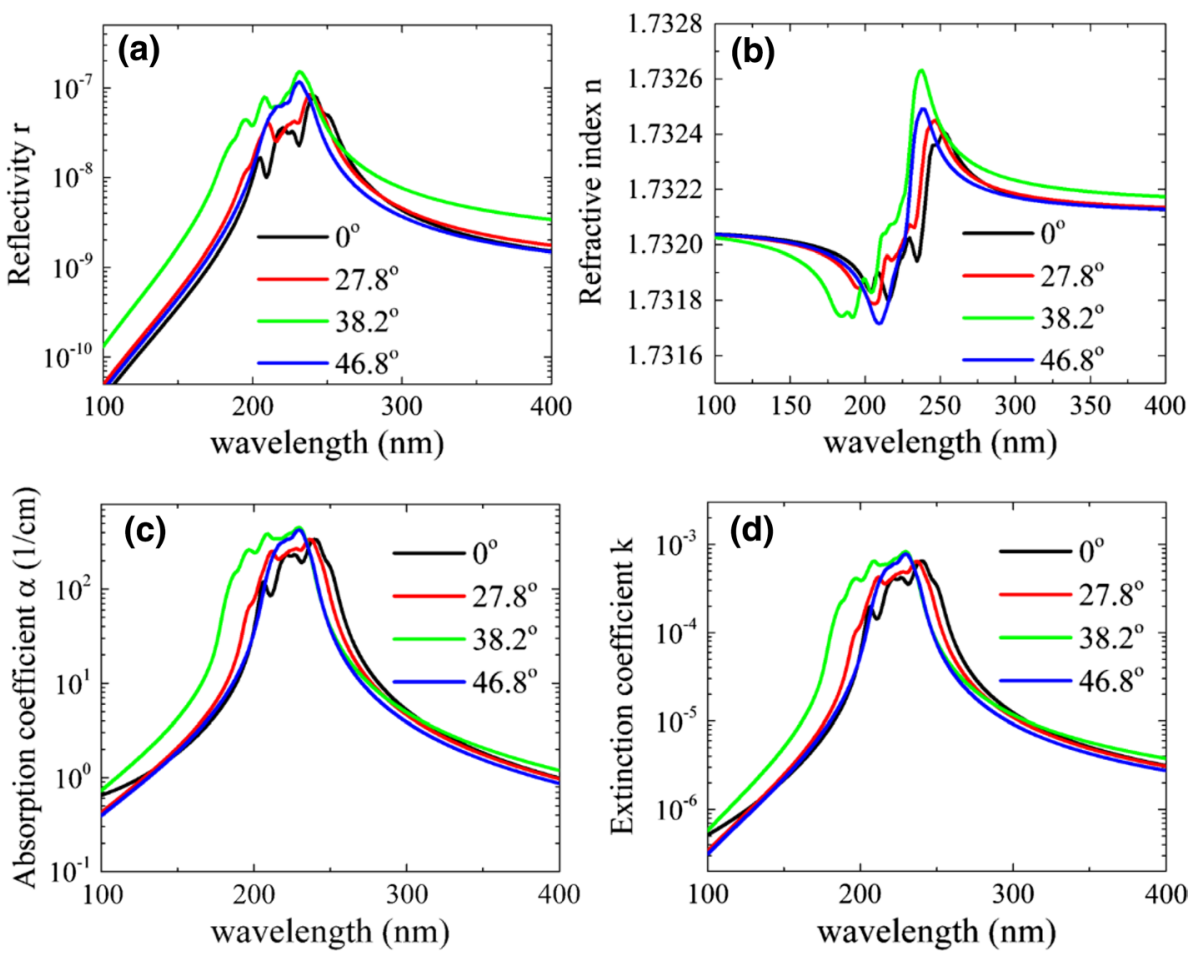

all cases. The peak amplitude values lie in the range from $10^{-8}$ to $10^{-7}$. In Fig. $6 \mathrm{~b}$, the refractive index values for all the cases are much smaller than the values for bulk GaN material. In Fig. 6c, the maximum values of the absorption coefficient in the spectrum for all the cases are around $400 \mathrm{~cm}^{-1}$. In Fig. 6d, the extinction coefficients in all cases follow the same trends as the absorption coefficient curves in terms of both the amplitude and wavelength. For the optical properties of the structure, a slight blue-shift is seen as a result of twisting. This occurs because the optical bandgap increases slightly as the rotation angle is increased. To explain these simulation results in terms of solid-state physics, a theoretical framework similar to that described in our previous paper [35] is utilized. Briefly, it is understood that the Lorentz model can be used to interpret the relations among all the above optical properties [36]. It is assumed that the bilayer structure and the incident optical light are sets of oscillating systems with harmonic resonant frequencies of $\omega_{0}$ and $\omega$, respectively. A high-absorption band appears when $\omega \approx \omega_{0}$. In the mismatched regions, this pseudomechanical theory applies in the interacting electron-photon system.

In optical applications, attenuation of the optical signal in a material is caused by absorption, scattering, and radiation of the optical power. In particular, the scattering loss occurs due to the refractive index caused by microscopic heterogeneities such as fluctuations in the material density or composition. The optical power loss due to scattering $l_{\mathrm{s}}$ is proportional to the eighth power of the refractive index $\left(l_{\mathrm{s}} \sim n^{8}\right)$ [37], so a small reduction of the refractive index can significantly reduce the scattering loss. Interestingly, the refractive index of bilayer $\mathrm{GaN}$ is much smaller $(\sim 1.73)$ compared with that of bulk GaN (2.3), resulting in greatly reduced scattering loss of the optical power, which could enable promising applications in optoelectronic devices. 
The optical power loss may be further reduced by the very low absorption of the bilayer structures simulated in this work, which is approximately three orders of magnitude lower compared with that of bulk GaN materials (reported to have an absorption coefficient of $\sim 10^{5} \mathrm{~cm}^{-1}$ [38]). Moreover, the bandgap of two-layer $\mathrm{GaN}$ is much wider than that of bulk materials, introducing prospective applications in power electronics. The results of this theoretical investigation using the first-principles method reveal that the bandgap of bilayer $\mathrm{GaN}$ can be further increased by the twisting effect. Such twisted bilayer structures bonded by the vdW force can already be achieved experimentally [39]. Although experiments on bilayer twisted $\mathrm{GaN}$ are not found, such experiments on other semiconductor materials predict that it should also be feasible for GaN.

\section{Conclusions}

The electronic and optical properties of bilayer $\mathrm{GaN}$ structures with rotation angles of $0^{\circ}, 27.8^{\circ}, 38.2^{\circ}$, and $46.8^{\circ}$ are simulated using density functional theory. According to the results of these first-principles simulations, the bandgap, absorption coefficient, extinction coefficient, reflectivity, and refractive index vary with the rotation angle. The structure formed of two vdW-bonded GaN monolayers is found to exhibit a wide bandgap, which can be further increased by twisting the two layers at certain optimized angles. It is also found that the refractive index of the bilayer structure is much smaller compared with that of its bulk form, greatly reducing the Rayleigh scattering of the optical power. The absorption coefficient is significantly reduced at all twisting angles including $0^{\circ}$. The optical absorption coefficient $\left(\sim 10^{2} \mathrm{~cm}^{-1}\right)$ calculated for bilayer $\mathrm{GaN}$ is much lower than that of bulk $\mathrm{GaN}\left(\sim 10^{5} \mathrm{~cm}^{-1}\right)$, reducing the power loss due to the absorption. Such bilayer structures thus offer the advantage of an increased optical bandgap and a reduced optical power loss due to the significant reduction of both the absorption coefficient and refractive index, making these bilayer GaN structures more suitable for monolithic integration of power electronic devices and optical devices. These results will be useful for future integrated low-loss optoelectronics applications.

Open Access This article is licensed under a Creative Commons Attribution 4.0 International License, which permits use, sharing, adaptation, distribution and reproduction in any medium or format, as long as you give appropriate credit to the original author(s) and the source, provide a link to the Creative Commons licence, and indicate if changes were made. The images or other third party material in this article are included in the article's Creative Commons licence, unless indicated otherwise in a credit line to the material. If material is not included in the article's Creative Commons licence and your intended use is not permitted by statutory regulation or exceeds the permitted use, you will need to obtain permission directly from the copyright holder. To view a copy of this licence, visit http://creativecommons.org/licenses/by/4.0/.

\section{References}

1. Avramescu, A., Lermer, T., Müller, J., Tautz, S., Queren, D., Lutgen, S., Strauß, U.: InGaN laser diodes with $50 \mathrm{~mW}$ output power emitting at $515 \mathrm{~nm}$. Appl. Phys. Lett. 95, 071103 (2009)

2. Khan, M.A., Kuznia, J.N., Olson, D.T., Hove, J.M.V., Blasingame, M., Reitz, L.F.: High-responsivity photoconductive ultraviolet sensors based on insulating single-crystal GaN epilayers. Appl. Phys. Lett. 60, 2917-2919 (1992)

3. Neufeld, C.J., Toledo, N.G., Cruz, S.C., Iza, M., DenBaars, S.P., Mishra, U.K.: High quantum efficiency InGaN/GaN solar cells with 2.95 eV band gap. Appl. Phys. Lett. 93, 143502 (2008)

4. Wang, X.H., Guo, L.W., Jia, H.Q., Xing, Z.G., Wang, Y., Pei, X.J., Zhou, J.M., Chen, H.: Control performance of a singlechip white light emitting diode by adjusting strain in InGaN underlying layer. Appl. Phys. Lett. 94, 111913 (2009)

5. Fan, Z.-Q., Jiang, X.-W., Chen, J., Luo, J.-W.: Improving performances of in-plane transition-metal dichalcogenide Schottky barrier field-effect transistors. ACS Appl. Mater. Interfaces 10, 19271-19277 (2018)

6. Fan, Z.-Q., Jiang, X.-W., Luo, J.-W., Jiao, L.-Y., Huang, R., Li, S.-S., Wang, L.-W.: In-plane Schottky-barrier field-effect transistors based on $1 \mathrm{~T} / 2 \mathrm{H}$ heterojunctions of transition-metal dichalcogenides. Phys. Rev. B 96, 165402 (2017)

7. Liu, Y.-Y., Li, B.-L., Chen, S.-Z., Jiang, X., Chen, K.-Q.: Effect of room temperature lattice vibration on the electron transport in graphene nanoribbons. Appl. Phys. Lett. 111, 133107 (2017)

8. Liu, Y.-Y., Zeng, Y.-J., Jia, P.-Z., Cao, X.-H., Jiang, X., Chen, K.-Q.: An efficient mechanism for enhancing the thermoelectricity of nanoribbons by blocking phonon transport in 2D materials. J. Phys. Condens. Matter 30, 275701 (2018)

9. Novoselov, K.S., Geim, A.K., Morozov, S.V., Jiang, D., Katsnelson, M.I., Grigorieva, I.V., Dubonos, S.V., Firsov, A.A.: Twodimensional gas of massless Dirac fermions in graphene. Nature 438, 197-200 (2005)

10. Novoselov, K.S., Geim, A.K., Morozov, S.V., Jiang, D., Zhang, Y., Dubonos, S.V., Grigorieva, I.V., Firsov, A.A.: Electric field effect in atomically thin carbon films. Science 306, 666-669 (2004)

11. Radisavljevic, B., Radenovic, A., Brivio, J., Giacometti, V., Kis, A.: Single-layer $\mathrm{MoS}_{2}$ transistors. Nat. Nanotechnol. 6, 147-150 (2011)

12. Ryder, C.R., Wood, J.D., Wells, S.A., Yang, Y., Jariwala, D., Marks, T.J., Schatz, G.C., Hersam, M.C.: Covalent functionalization and passivation of exfoliated black phosphorus via aryl diazonium chemistry. Nat. Chem. 8, 597-602 (2016)

13. Song, L., Ci, L., Lu, H., Sorokin, P.B., Jin, C., Ni, J., Kvashnin, A.G., Kvashnin, D.G., Lou, J., Yakobson, B.I., Ajayan, P.M.: Large scale growth and characterization of atomic hexagonal boron nitride layers. Nano Lett. 10, 3209-3215 (2010)

14. Wang, Y., Zhou, W.-X., Huang, L., Xia, C., Tang, L.-M., Deng, H.-X., Li, Y., Chen, K.-Q., Li, J., Wei, Z.: Light induced double 'on' state anti-ambipolar behavior and self-driven photoswitching in $p$ - $\mathrm{WSe}_{2} / n-\mathrm{SnS}_{2}$ heterostructures. 2D Mater. 4, 025097 (2017)

15. Wu, S., Ross, J.S., Liu, G.-B., Aivazian, G., Jones, A., Fei, Z., Zhu, W., Xiao, D., Yao, W., Cobden, D., Xu, X.: Electrical tuning of valley magnetic moment through symmetry control in bilayer $\mathrm{MoS}_{2}$. Nat. Phys. 9, 149-153 (2013) 
16. Deng, S., Li, L., Li, M.: Stability of direct band gap under mechanical strains for monolayer $\mathrm{MoS}_{2}, \mathrm{MoSe}_{2}, \mathrm{WS}_{2}$ and $\mathrm{WSe}_{2}$. Physica E 101, 44-49 (2018)

17. Şahin, H., Cahangirov, S., Topsakal, M., Bekaroglu, E., Akturk, E., Senger, R.T., Ciraci, S.: Monolayer honeycomb structures of group-IV elements and III-V binary compounds: first-principles calculations. Phys. Rev. B 80, 155453 (2009)

18. Chen, Q., Hu, H., Chen, X., Wang, J.: Tailoring band gap in GaN sheet by chemical modification and electric field: ab initio calculations. Appl. Phys. Lett. 98, 053102 (2011)

19. Xiao, W.-Z., Wang, L.-L., Xu, L., Wan, Q., Pan, A.-L., Deng, H.-Q.: Ferromagnetic and metallic properties of the semihydrogenated GaN sheet. Phys. Status Solidi (B) 248, 1442-1445 (2011)

20. $\mathrm{Mu}, \mathrm{Y} .:$ Chemical functionalization of $\mathrm{GaN}$ monolayer by adatom adsorption. J. Phys. Chem. C 119, 20911-20916 (2015)

21. Tang, W., Sun, M., Yu, J., Chou, J.-P.: Magnetism in non-metal atoms adsorbed graphene-like gallium nitride monolayers. Appl. Surf. Sci. 427, 609-612 (2018)

22. Zhang, X., Jin, L., Dai, X., Chen, G., Liu, G.: Two-Dimensional $\mathrm{GaN}$ : an excellent electrode material providing fast ion diffusion and high storage capacity for Li-Ion and Na-Ion batteries. ACS Appl. Mater. Interfaces 10, 38978-38984 (2018)

23. Tian, J., Liu, L., Xia, S., Diao, Y., Lu, F.: Optoelectronic properties of two-dimensional GaN adsorbed with $\mathrm{H}, \mathrm{N}$ and $\mathrm{O}$ : a firstprinciple study. Phys. Lett. A 383, 3018-3024 (2019)

24. Gao, H., Ye, H., Yu, Z., Zhang, Y., Liu, Y., Li, Y.: Point defects and composition in hexagonal group-III nitride monolayers: a first-principles calculation. Superlattices Microstruct. 112, 136$142(2017)$

25. González, R., López-Pérez, W., González-García, Á., MorenoArmenta, M.G., González-Hernández, R.: Vacancy charged defects in two-dimensional GaN. Appl. Surf. Sci. 433, 1049-1055 (2018)

26. Li, J., Liu, H.: Magnetism investigation of GaN monolayer doped with group VIII B transition metals. J. Mater. Sci. 53, 1598615994 (2018)

27. Zhao, Q., Xiong, Z., Luo, L., Sun, Z., Qin, Z., Chen, L., Wu, N.: Design of a new two-dimensional diluted magnetic semiconductor: Mn-doped GaN monolayer. Appl. Surf. Sci. 396, 480-483 (2017)

28. Al Balushi, Z.Y., Wang, K., Ghosh, R.K., Vilá, R.A., Eichfeld, S.M., Caldwell, J.D., Qin, X., Lin, Y.-C., DeSario, P.A., Stone, G.,
Subramanian, S., Paul, D.F., Wallace, R.M., Datta, S., Redwing, J.M., Robinson, J.A.: Two-dimensional gallium nitride realized via graphene encapsulation. Nat. Mater. 15, 1166-1171 (2016)

29. Koratkar, N.A.: Two-dimensional gallium nitride. Nat. Mater. 15, 1153-1154 (2016)

30. Chen, Y., Liu, K., Liu, J., Lv, T., Wei, B., Zhang, T., Zeng, M., Wang, Z., Fu, L.: Growth of 2D GaN single crystals on liquid metals. J. Am. Chem. Soc. 140, 16392-16395 (2018)

31. Cao, Y., Fatemi, V., Demir, A., Fang, S., Tomarken, S.L., Luo, J.Y., Sanchez-Yamagishi, J.D., Watanabe, K., Taniguchi, T., Kaxiras, E., Ashoori, R.C., Jarillo-Herrero, P.: Correlated insulator behaviour at half-filling in magic-angle graphene superlattices. Nature 556, 80-84 (2018)

32. Cao, Y., Fatemi, V., Fang, S., Watanabe, K., Taniguchi, T., Kaxiras, E., Jarillo-Herrero, P.: Unconventional superconductivity in magic-angle graphene superlattices. Nature 556, 43-50 (2018)

33. Le, N.B., Huan, T.D., Woods, L.M.: Interlayer interactions in van der Waals heterostructures: electron and phonon properties. ACS Appl. Mater. Interfaces 8, 6286-6292 (2016)

34. Deng, S., Li, L., Rees, P.: Graphene/MoXY heterostructures adjusted by interlayer distance, external electric field, and strain for tunable devices. ACS Appl. Nano Mater. 2, 3977-3988 (2019)

35. Deng, S., Zhang, Y., Li, L.: Study on electronic and optical properties of the twisted and strained MoS2/PtS2 heterogeneous interface. Appl. Surf. Sci. 476, 308-316 (2019)

36. Wooden, F.: Optical Properties of Solids. Academic, New York (1972)

37. Okamoto, K.: Fundamentals of Optical Waveguides, 2nd edn. Academic, Burlington (2006)

38. Muth, J.F., Lee, J.H., Shmagin, I.K., Kolbas, R.M., Casey Jr., H.C., Keller, B.P., Mishra, U.K., DenBaars, S.P.: Absorption coefficient, energy gap, exciton binding energy, and recombination lifetime of GaN obtained from transmission measurements. Appl. Phys. Lett. 71, 2572-2574 (1997)

39. Novoselov, K.S., Mishchenko, A., Carvalho, A., Castro Neto, A.H.: 2D materials and van der Waals heterostructures. Science 353, aac9439 (2016)

Publisher's Note Springer Nature remains neutral with regard to jurisdictional claims in published maps and institutional affiliations. 